\title{
Bifurcation Analysis and Chaos Control in a Discrete Epidemic System
}

\author{
Wei Tan, ${ }^{1,2}$ Jianguo Gao, ${ }^{1}$ and Wenjun Fan ${ }^{1}$ \\ ${ }^{1}$ Department of Mathematics and Information Science, Beifang University of Nationalities, Yinchuan, Ningxia 750021, China \\ ${ }^{2}$ Department of Mathematics and Statistics, Zhoukou Normal University, Zhoukou, Henan 466001, China \\ Correspondence should be addressed to Jianguo Gao; gaojguo@163.com
}

Received 14 June 2015; Accepted 9 August 2015

Academic Editor: Garyfalos Papashinopoulos

Copyright (c) 2015 Wei Tan et al. This is an open access article distributed under the Creative Commons Attribution License, which permits unrestricted use, distribution, and reproduction in any medium, provided the original work is properly cited.

\begin{abstract}
The dynamics of discrete SI epidemic model, which has been obtained by the forward Euler scheme, is investigated in detail. By using the center manifold theorem and bifurcation theorem in the interior $R_{+}^{2}$, the specific conditions for the existence of flip bifurcation and Neimark-Sacker bifurcation have been derived. Numerical simulation not only presents our theoretical analysis but also exhibits rich and complex dynamical behavior existing in the case of the windows of period-1, period-3, period-5, period-6, period-7, period-9, period-11, period-15, period-19, period-23, period-34, period-42, and period-53 orbits. Meanwhile, there appears the cascade of period-doubling 2, 4, 8 bifurcation and chaos sets from the fixed point. These results show the discrete model has more richer dynamics compared with the continuous model. The computations of the largest Lyapunov exponents more than 0 confirm the chaotic behaviors of the system $x \rightarrow x+\delta[r N(1-N / K)-\beta(x y / N)-(\mu+m) x], y \rightarrow y+\delta[\beta(x y / N)-(\mu+d) y]$. Specifically, the chaotic orbits at an unstable fixed point are stabilized by using the feedback control method.
\end{abstract}

\section{Introduction}

Within the field of epidemic theory, both the continuous-time model and the discrete-time model were described by the different equations. The continuous-time epidemic models have been widely investigated by many researchers (e.g., [1-10]). A Leslie-Gower predator-prey model with disease in prey incorporating a prey refuge was investigated in [7]. A ratiodependent predator-prey model with Allee effect and disease in prey was investigated in [8]. Meanwhile, plenty of scholars used some discretization scheme to the continuous-time systems to study the consistency, convergence, permanence, and stability of the discrete system in [11-16]. In the recent years, there appear to be a number of articles about the flip bifurcation, fold bifurcation, and pitchfork bifurcation and in the sense of Marottos chaos of the discrete system which were presented in [17-28]. Both a discrete-time predator-prey system without Allee effect and a discrete-time predator-prey system with Allee effect were studied in [22, 23]. Dynamics of a system of three interacting populations with Allee effects and stocking was researched in [26]. Stability analysis and rich oscillation patterns in discrete-time FitzHugh-Nagumo excitable system with delayed coupling were studied in [24].

In this paper by using the center manifold theorem and bifurcation theorem, the local stability and bifurcation behaviors of a simple SI epidemic model with surprising dynamic is investigated in detail. The paper is organized as follows. Section 2 gives the specific conditions for existence of flip bifurcation and Neimark-Sacker bifurcation. Section 3 analyzes the two bifurcations by choosing the time step size $\delta$ as the parameter of the bifurcation. Section 4 verifies the theoretical analysis and displays the complex and surprising dynamics. In Section 5, chaos approaching to the unable fixed point is controlled by using the feedback control method.

A simple SI epidemic model with surprising dynamic was showed in [1] as

$$
\begin{aligned}
& \dot{S}(t)=r N\left(1-\frac{N}{K}\right)-\frac{\beta S I}{N}-(\mu+m) S, \\
& \dot{I}(t)=\frac{\beta S I}{N}-(\mu+d) I,
\end{aligned}
$$


where $N=S+I$ is the total population size; the birth process incorporates density dependent effects through a logistic equation with intrinsic growth rate $r$ and the carrying capacity $K$, and other parameters are positive constant.

It is obvious that system (1) is a two-dimensional nonlinear ODE, where it is an impossible task to obtain its analytical solutions to study its qualitative properties including the global stability, so it is necessary to solve numerical solutions or approximate solutions of system (1) according to different discrete difference schemes. Therefore, in order to study the dynamics behavior of system (1), we will focus on the complex dynamical behaviors of a simple discrete SI epidemic model approached by the forward Euler scheme.

In this paper, we apply the forward Euler scheme to system (1) to get the following form:

$$
\begin{aligned}
& x \longrightarrow x+\delta\left[r N\left(1-\frac{N}{K}\right)-\beta \frac{x y}{N}-(\mu+m) x\right], \\
& y \longrightarrow y+\delta\left[\beta \frac{x y}{N}-(\mu+d) y\right],
\end{aligned}
$$

where $\delta$ is the step size.

\section{Stability of Fixed Points}

The fixed points of system (2) are $A(r N(1-N / K) /(\mu+m), 0)$ and $B\left(x^{*}, y^{*}\right)$, where $x^{*}, y^{*}$ satisfy

$$
\begin{aligned}
r N\left(1-\frac{N}{K}\right)-\frac{\beta x y}{N}-(\mu+m) x & =0, \\
\frac{\beta x y}{N}-(\mu+d) y & =0 .
\end{aligned}
$$

The Jacobian matrix of (2) at any point $(x, y)$ is written as

$$
J=\left[\begin{array}{cc}
1-\delta\left(\frac{\beta}{N} y+\mu+m\right) & -\delta\left(\frac{\beta}{N} x\right) \\
\delta\left(\frac{\beta}{N} y\right) & 1+\delta\left(\frac{\beta}{N} x-\mu-d\right)
\end{array}\right] .
$$

The characteristic equation of Jacobian matrix (4) is given by

$$
F(\lambda)=\lambda^{2}+p(x, y) \lambda+q(x, y)=0,
$$

where

$$
\begin{aligned}
& p(x, y)=-2+\delta\left[\frac{\beta}{N}(y-x)+2 \mu+d+m\right], \\
& q(x, y) \\
& =\left[1-\delta\left(\frac{\beta}{N} y+\mu+m\right)\right]\left[1+\delta\left(\frac{\beta}{N} x-\mu-d\right)\right] \\
& \quad+\delta^{2} \frac{\beta^{2}}{N^{2}} x y .
\end{aligned}
$$

Let us give a lemma as similar as in [21].

Lemma 1. Suppose $\lambda_{1}$ and $\lambda_{2}$ are two roots of $F(\lambda)=0$; the local stability of the positive point can be determined by the modules of $\left|\lambda_{1}\right|$ and $\left|\lambda_{2}\right|$. (i) If $\left|\lambda_{1}\right| \leqslant 1$ and $\left|\lambda_{2}\right| \leqslant 1$, then not only is $B\left(X^{*}, Y^{*}\right) a$ sink, but also it is locally asymptotically stable.

(ii) If $\left|\lambda_{1}\right| \geqslant 1$ and $\left|\lambda_{2}\right| \geqslant 1$, then not only is $B\left(X^{*}, Y^{*}\right) a$ source, but also it is unstable.

(iii) If $\left|\lambda_{1}\right| \leqslant 1$ and $\left|\lambda_{2}\right| \geqslant 1\left(\left|\lambda_{1}\right| \geqslant 1\right.$ and $\left.\left|\lambda_{2}\right| \leqslant 1\right)$, then $B\left(X^{*}, Y^{*}\right)$ is a saddle.

(iv) If $\left|\lambda_{1}\right|=1$ or $\left|\lambda_{2}\right|=1$, then $B\left(X^{*}, Y^{*}\right)$ is nonhyperbolic.

In order to discuss the stability of the fixed point of system (2), the following lemma is also needed which can be easily proved by the relation between roots and coefficient of a quadratic equation [21].

Lemma 2. Let $F(\lambda)=\lambda^{2}+p \lambda+q$. Suppose that $F(1)>$ $0(F(1)<0) ; \lambda_{1}$ and $\lambda_{2}$ are root of $F(\lambda)=0$. Then

(i) $\left|\lambda_{1}\right| \leqslant 1$ and $\left|\lambda_{2}\right| \leqslant 1$ if $F(-1)>0$ and $q<1$;

(ii) $\left|\lambda_{1}\right| \leqslant 1$ and $\left|\lambda_{2}\right| \geqslant 1\left(\left|\lambda_{1}\right| \geqslant 1\right.$ and $\left.\left|\lambda_{2}\right| \leqslant 1\right)$ if and only if $F(-1)<0$;

(iii) $\left|\lambda_{1}\right| \geqslant 1$ and $\left|\lambda_{2}\right| \geqslant 1$ if and only if $F(-1)>0$ and $q>1$

(iv) $\left|\lambda_{1}\right|=1$ and $\left|\lambda_{2}\right| \neq 1$ if and only if $F(-1)=0$ and $p \neq 0,2$

(v) $\left|\lambda_{1}\right|=1$ or $\left|\lambda_{2}\right|=1$ and $\lambda_{1}$ and $\lambda_{2}$ are complex conjugate if and only if $p^{2}-4 q<0$ and $q=1$.

Proposition 3. The eigenvalues of the fixed point $A(r N(1-$ $N / K) /(\mu+m), 0)$ are $\lambda_{1}=1-\delta(\mu+m), \lambda_{2}=1+\delta[\beta r(1-$ $N / K) /(\mu+m)-\mu-m]$.

(i) $A(r N(1-N / K) /(\mu+m), 0)$ is sink if $(\mu+m)(\mu+d)>$ $\beta r(1-N / k)$ and $0<\delta<\min \{2 /(\mu+m), 2((\mu+d)(\mu+$ $m) / \beta r(1-N / k))\}$.

(ii) $A(r N(1-N / K) /(\mu+m), 0)$ is source if $(\mu+m)(\mu+d)>$ $\beta r(1-N / k)$ and $\delta>\max \{2 /(\mu+m), 2((\mu+d)(\mu+$ $m) / \beta r(1-N / k))\}$.

(iii) $A(r N(1-N / K) /(\mu+m), 0)$ is nonhyperbolic if $(\mu+$ $m)(\mu+d)>\beta r(1-N / k)$ and $\delta=2 /(\mu+m)$ or $\delta=$ $2((\mu+d)(\mu+m) / \beta r(1-N / k))$.

(iv) $A(r N(1-N / K) /(\mu+m), 0)$ is a saddle, except for that values of parameters which (i) to (iii).

Let

$$
\begin{aligned}
F_{A} & =\{(r, N, K, \beta, \mu, m, \delta):(\mu+m)(\mu+d) \\
& >\beta r\left(1-\frac{N}{k}\right), \delta=\frac{2}{\mu+m}, \delta \\
& \left.\neq 2 \frac{(\mu+d)(\mu+m)}{\beta r(1-N / k)}, r, N, K, \beta, \mu, m, \delta>0\right\} .
\end{aligned}
$$

If term (iii) of Proposition 3 holds, then one of the eigenvalues of the fixed point $A(r N(1-N / K) /(\mu+m), 0)$ is -1 and the magnitude of the other is not equal to 1 . The point $A(r N(1-N / K) /(\mu+m), 0)$ undergoes flip bifurcation when the parameter changes in small neighborhood of $F_{A}$. 
The characteristic equation of the Jacobian matrix $J$ of Let system (2) at the positive $B\left(X^{*}, Y^{*}\right)$ is written as

$$
\lambda^{2}+p\left(x^{*}, y^{*}\right) \lambda+q\left(x^{*}, y^{*}\right)=0
$$

where

$$
\begin{aligned}
& p\left(x^{*}, y^{*}\right)=-2-\delta E, \\
& q\left(x^{*}, y^{*}\right)=1+\delta E+\delta^{2} F, \\
& E=\frac{\beta}{N}\left(x^{*}-y^{*}\right)-2 \mu-d-m, \\
& F \quad\left[-\left(\frac{\beta}{N} y^{*}+\mu+m\right)\right]\left[\left(\frac{\beta}{N} x^{*}-\mu-d\right)\right] \\
& \quad+\frac{\beta^{2}}{N^{2}} x^{*} y^{*} .
\end{aligned}
$$

Let

$$
F(\lambda)=\lambda^{2}-(2+\delta E) \lambda+1+\delta E+\delta^{2} F
$$

Therefore,

$$
\begin{gathered}
F(1)=\delta^{2} F \\
F(-1)=4+2 \delta E+\delta^{2} F .
\end{gathered}
$$

According to Lemma 2, we get the following proposition.

Proposition 4. There exists the fixed point $B\left(x^{*}, y^{*}\right)$ for every possible parameter:

(i) $B\left(x^{*}, y^{*}\right)$ is a sink if satisfying

(i.1) $E^{2}-4 F \geqslant 0$ and $0 \leqslant \delta<\left(-E-\sqrt{E^{2}-4 F}\right) / F$;

(i.2) $E^{2}-4 F<0$ and $0 \leqslant \delta<-E / F$;

(ii) $B\left(x^{*}, y^{*}\right)$ is a source if satisfying

(ii.1) $E^{2}-4 F \geqslant 0$ and $\delta>\left(-E+\sqrt{E^{2}-4 F}\right) / F$;

(ii.2) $E^{2}-4 F<0$ and $\delta>-E / F$;

(iii) $B\left(x^{*}, y^{*}\right)$ is nonhyperbolic if satisfying

(iii.1) $E^{2}-4 F \geqslant 0$ and $\delta=\left(-E \mp \sqrt{E^{2}-4 F}\right) / F$;

(iii.2) $E^{2}-4 F<0$ and $\delta=-E / F$;

(iv) $B\left(x^{*}, y^{*}\right)$ is a saddle, except for that values of parameters which (i) to (iii). If (iii.1) holds, then $\lambda_{1}=-1$ and $\left|\lambda_{2}\right| \neq 1$.

$$
\begin{aligned}
F_{B_{1}} & =\left\{(r, N, K, \beta, \mu, m, \delta): E^{2}-4 F \geqslant 0, \delta\right. \\
& \left.=\frac{-E-\sqrt{E^{2}-4 F}}{F}, r, N, K, \beta, \mu, m, \delta>0\right\}, \\
F_{B_{2}} & =\left\{(r, N, K, \beta, \mu, m, \delta): E^{2}-4 F \geqslant 0, \delta\right. \\
& \left.=\frac{-E+\sqrt{E^{2}-4 F}}{F}, r, N, K, \beta, \mu, m, \delta>0\right\} .
\end{aligned}
$$

If (iii.2) holds, then $\lambda_{1}$ and $\lambda_{2}$ are a pair of complex conjugate numbers and $\lambda_{1}=-1$ and $\left|\lambda_{2}\right|=1$.

Let

$$
\begin{aligned}
E_{B_{1}} & =\left\{(r, N, K, \beta, \mu, m, \delta): E^{2}-4 F<0, \delta\right. \\
& \left.=-\frac{E}{F}, r, N, K, \beta, \mu, m, \delta>0\right\} .
\end{aligned}
$$

\section{Bifurcation Analysis on the Positive $B\left(x^{*}, y^{*}\right)$}

In this section, we will investigate the flip bifurcation and Neimark-Sacker bifurcation corresponding to the positive fixed point.

3.1. Flip Bifurcation. Consider system (2) with arbitrary parameter $(r, N, K, \beta, \mu, m, \delta) \in F_{B_{1}}$, then

$$
\begin{aligned}
& x \longrightarrow x+\delta_{1}\left[r N\left(1-\frac{N}{K}\right)-\beta \frac{x y}{N}-(\mu+m) x\right], \\
& y \longrightarrow y+\delta_{1}\left[\beta \frac{x y}{N}-(\mu+d) y\right]
\end{aligned}
$$

where

$$
\begin{aligned}
\delta_{1} & =\frac{-E-\sqrt{E^{2}-4 F}}{F}, \\
\lambda_{1} & =-1, \\
\left|\lambda_{2}\right| & \neq 1 .
\end{aligned}
$$

Now, give a perturbation of model (14) as follows:

$$
\begin{aligned}
x & \longrightarrow x \\
& +\left(\delta_{1}+\delta^{*}\right)\left[r N\left(1-\frac{N}{K}\right)-\beta \frac{x y}{N}-(\mu+m) x\right], \\
y & \longrightarrow y+\left(\delta_{1}+\delta^{*}\right)\left[\beta \frac{x y}{N}-(\mu+d) y\right],
\end{aligned}
$$

where $\delta^{*} \ll 1$ is a limited perturbation parameter. 
Let $X=x^{*}-x, Y=y-y^{*}$. After the transformation of the fixed $B\left(x^{*}, y^{*}\right)$ to $(0,0)$, as follows:

$$
\begin{aligned}
X \longrightarrow & a_{11} X+a_{12} Y+a_{13} X Y+a_{14} \delta^{*} X+a_{15} \delta^{*} Y \\
& +a_{16} \delta^{*} X Y, \\
Y \longrightarrow & a_{21} X+a_{22} Y+a_{23} X Y+a_{24} \delta^{*} X+a_{25} \delta^{*} Y \\
& +a_{26} \delta^{*} X Y,
\end{aligned}
$$

where

$$
\begin{aligned}
& a_{11}=1-\delta_{1}\left[\frac{\beta}{N} y^{*}+\mu+m\right], \\
& a_{12}=-\delta_{1} \frac{\beta}{N} x^{*}, \\
& a_{13}=-\delta_{1} \frac{\beta}{N}, \\
& a_{14}=-\left(\frac{\beta}{N} y^{*}+\mu+m\right), \\
& a_{15}=-\frac{\beta}{N} x^{*}, \\
& a_{16}=-\frac{\beta}{N}, \\
& a_{21}=\delta_{1} \frac{\beta}{N} y^{*}, \\
& a_{25}=\frac{\beta}{N} x^{*}-\mu-d, \\
& a_{22}=1+\delta_{1}\left(\frac{\beta}{N} x^{*}-\mu-d\right), \\
& a_{23}=\frac{\beta}{N} \delta_{1}, \\
& a_{24}
\end{aligned}
$$

and $\delta=\delta_{1}$.

Let

$$
T=\left[\begin{array}{cc}
a_{12} & a_{12} \\
-1-a_{11} & \lambda_{2}-a_{11}
\end{array}\right] \text {. }
$$

Consider the following translation:

$$
\left[\begin{array}{l}
X \\
Y
\end{array}\right]=T\left[\begin{array}{l}
u \\
v
\end{array}\right]
$$

Taking $T^{-1}$ on both sides of (17), obtain

$$
\left[\begin{array}{l}
u \\
v
\end{array}\right]=\left[\begin{array}{cc}
-1 & 0 \\
0 & \lambda_{2}
\end{array}\right]\left[\begin{array}{l}
u \\
v
\end{array}\right]+\left[\begin{array}{l}
f\left(X, Y, \delta^{*}\right) \\
g\left(X, Y, \delta^{*}\right)
\end{array}\right],
$$

where

$$
\begin{aligned}
& f\left(X, Y, \delta^{*}\right)=\left(\frac{1}{a_{12}\left(\lambda_{2}+1\right)}\right) \\
& \cdot\left\{\left[a_{13}\left(\lambda_{2}-a_{11}\right)-a_{12} a_{23}\right] X Y\right. \\
& +\left[\left(\lambda_{2}-a_{11}\right) a_{14}-a_{12} a_{24}\right] \delta^{*} X \\
& \quad+\left[\left(\lambda_{2}-a_{11}\right) a_{15}-a_{12} a_{25}\right] \delta^{*} Y \\
& \left.\quad+\left[\left(\lambda_{2}-a_{11}\right) a_{16}-a_{12} a_{26}\right] \delta^{*} X Y\right\}, \\
& g\left(X, Y, \delta^{*}\right)=\left(\frac{1}{a_{12}\left(\lambda_{2}+1\right)}\right) \\
& \quad+\left\{\left[a_{13}\left(1+a_{11}\right)+a_{12} a_{23}\right] X Y\right. \\
& +\left[\left(1+a_{11}\right) a_{14}+a_{12} a_{24}\right] \delta^{*} X \\
& +\left[\left(1+a_{11}\right) a_{15}+a_{12} a_{25}\right] \delta^{*} Y \\
& \left.+\left[\left(1+a_{11}\right) a_{16}+a_{12} a_{26}\right] \delta^{*} X Y\right\}, \\
& \quad X=a_{12}(u+v), Y=-\left(1+a_{11}\right) u+\left(\lambda_{2}-a_{11}\right) v .
\end{aligned}
$$

Apply the center manifold theorem to (17) at the origin in the limited neighborhood of $\delta^{*}=0$. The center manifold $W^{c}(0,0,0)$ can be approximately presented as

$$
\begin{aligned}
& W^{c}(0,0,0)=\left\{\left(u, v, \delta^{*}\right) \in R^{3} \mid v=h\left(u, \delta^{*}\right), h(0,0)\right. \\
& \quad=0, \operatorname{Dh}(0,0)=0\},
\end{aligned}
$$

for $u, \delta^{*}$ sufficiently small.

We assume a center manifold of the form

$$
h\left(u, \delta^{*}\right)=a_{1} u^{2}+a_{2} \delta^{*} u+0\left(\left(|u|+\left|\delta^{*}\right|\right)^{3}\right),
$$

which must satisfy

$$
\begin{aligned}
N\left(h\left(u, \delta^{*}\right)\right)= & h\left(-u+f\left(u, h\left(u, \delta^{*}\right)\right), \delta^{*}\right) \\
& -\lambda_{2} h\left(u, \delta^{*}\right)-g\left(u, h\left(u, \delta^{*}\right), \delta^{*}\right) \\
= & 0 .
\end{aligned}
$$

By the simple calculations, obtain

$a_{1}=\frac{\left[a_{13}\left(1+a_{11}\right)+a_{12} a_{23}\right]\left(1+a_{11}\right)}{\lambda_{2}^{2}-1}$

$a_{2}$

$=\frac{-\left[\left(1+a_{11}\right) a_{14}+a_{12} a_{24}\right] a_{12}+\left[\left(1+a_{11}\right) a_{15}+a_{12} a_{25}\right]\left(1+a_{11}\right)}{a_{12}\left(1+\lambda_{2}\right)^{2}}$. 
Consider the map restricted to the center manifold $W^{c}(0,0,0)$ :

$$
\begin{aligned}
f: \longrightarrow & -u+\frac{h_{1}}{a_{12}\left(\lambda_{2}+1\right)} u^{2}+\frac{h_{2}}{a_{12}\left(\lambda_{2}+1\right)} u \delta^{*} \\
& +\frac{h_{3}}{a_{12}\left(\lambda_{2}+1\right)} u^{3}+\frac{h_{4}}{a_{12}\left(\lambda_{2}+1\right)} \delta^{*} u^{2} \\
& +\frac{h_{5}}{a_{12}\left(\lambda_{2}+1\right)}\left(\delta^{*}\right)^{2} u,
\end{aligned}
$$

where

$$
\begin{aligned}
h_{1}= & -\left[a_{13}\left(\lambda_{2}-a_{11}\right)-a_{12} a_{23}\right] a_{12}\left(1+a_{11}\right), \\
h_{2}= & {\left[\left(\lambda_{2}-a_{11}\right) a_{14}-a_{12} a_{24}\right] a_{12} } \\
& -\left[\left(\lambda_{2}-a_{11}\right) a_{15}-a_{12} a_{25}\right]\left(1+a_{11}\right), \\
h_{3}= & {\left[a_{13}\left(\lambda_{2}-a_{11}\right)-a_{12} a_{23}\right] a_{12} a_{1}\left[\lambda_{2}-2 a_{11}-1\right], } \\
h_{4}= & {\left[a_{13}\left(\lambda_{2}-a_{11}\right)-a_{12} a_{23}\right] a_{12} a_{2}\left[\lambda_{2}-2 a_{11}-1\right] } \\
& +\left[\left(\lambda_{2}-a_{11}\right) a_{14}-a_{12} a_{24}\right] a_{12} a_{1} \\
& +\left[\left(\lambda_{2}-a_{11}\right) a_{15}-a_{12} a_{25}\right] a_{1}\left(\lambda_{2}-a_{11}\right) \\
& -\left[\left(\lambda_{2}-a_{11}\right) a_{16}-a_{12} a_{26}\right] a_{12}\left(1+a_{11}\right), \\
h_{5}= & {\left[\left(\lambda_{2}-a_{11}\right) a_{14}-a_{12} a_{24}\right] a_{12} a_{2} } \\
& +\left[\left(\lambda_{2}-a_{11}\right) a_{15}-a_{12} a_{25}\right]\left(\lambda_{2}-a_{11}\right) a_{2}, \\
L= & a_{12}\left(\lambda_{2}+1\right) .
\end{aligned}
$$

According to flip bifurcation, we get

$$
\begin{aligned}
& l_{1}=\left(\frac{\partial^{2} f}{\partial u \partial \delta^{*}}+\frac{1}{2} \frac{\partial f}{\partial \delta^{*}} \frac{\partial^{2} f}{\partial u^{2}}\right) \mid(0,0), \\
& l_{2}=\left(\frac{1}{6} \frac{\partial^{3} f}{\partial u^{3}}+\left(\frac{1}{2} \frac{\partial^{2} f}{\partial u^{2}}\right)^{2}\right) \mid(0,0) .
\end{aligned}
$$

After simple calculations, we obtain

$$
\begin{aligned}
& l_{1}=\frac{h_{2}}{L}+\frac{h_{1}^{2}}{L^{2}}, \\
& l_{2}=\frac{h_{3}}{L}+\frac{h_{1}^{2}}{L^{2}} .
\end{aligned}
$$

Theorem 5. If $\alpha_{2} \neq 0$, system (2) will undergo flip bifurcation at the positive point $B\left(x^{*}, y^{*}\right)$. Also the period-2 orbits that bifurcate from fixed $B\left(x^{*}, y^{*}\right)$ are stable (resp., unstable) if $\alpha_{2}>0$ (resp., $\left.\alpha_{2}<0\right)$.

3.2. Neimark-Sacker Bifurcation. Consider model (2) with arbitrary parameter $(r, N, K, \beta, \mu, m, \delta) \in E_{B_{1}}$, which is described by

$$
\begin{aligned}
& x \longrightarrow x+\delta_{2}\left[r N\left(1-\frac{N}{K}\right)-\beta \frac{x y}{N}-(\mu+m) x\right], \\
& y \longrightarrow y+\delta_{2}\left[\beta \frac{x y}{N}-(\mu+d) y\right] .
\end{aligned}
$$

$B\left(x^{*}, y^{*}\right)$ is the fixed point of model (2), which is given by (3) and $\delta_{2}=-E / F$

We consider the perturbation of model (31) as follows:

$$
\begin{aligned}
x & \longrightarrow x \\
& +\left(\delta_{2}+\delta^{\star}\right)\left[r N\left(1-\frac{N}{K}\right)-\beta \frac{x y}{N}-(\mu+m) x\right], \\
y & \longrightarrow y+\left(\delta_{2}+\delta^{\star}\right)\left[\beta \frac{x y}{N}-(\mu+d) y\right],
\end{aligned}
$$

where $\delta^{\star} \ll 1$ is a limited perturbation parameter.

Let $X=x-x^{*}, Y=y-y^{*}$. After the transformation of the fixed $B\left(x^{*}, y^{*}\right)$ to $(0,0)$, as follows:

$$
\begin{aligned}
& X \longrightarrow b_{11} X+b_{12} Y+b_{13} X Y, \\
& Y \longrightarrow b_{21} X+b_{22} Y+b_{23} X Y,
\end{aligned}
$$

where

$$
\begin{aligned}
& b_{11}=1-\left(\delta_{2}+\delta^{\star}\right)\left(\frac{\beta}{N} y^{*}+\mu+m\right), \\
& b_{12}=-\left(\delta_{2}+\delta^{\star}\right) \frac{\beta}{N} x^{*}, \\
& b_{13}=-\left(\delta_{2}+\delta^{\star}\right) \frac{\beta}{N}, \\
& b_{21}=\left(\delta_{2}+\delta^{\star}\right) \frac{\beta}{N} y^{*}, \\
& b_{22}=1+\left(\delta_{2}+\delta^{\star}\right)\left(\frac{\beta}{N} x^{*}-\mu-d\right), \\
& b_{23}=\left(\delta_{2}+\delta^{\star}\right) \frac{\beta}{N},
\end{aligned}
$$

and $\delta=\delta_{2}$.

The characteristic equation of model (31) at $B\left(x^{*}, y^{*}\right)$ is as follows:

$$
\lambda^{2}+p\left(\delta^{\star}\right) \lambda+q\left(\delta^{\star}\right)=0 .
$$

Since the parameter $\left(r, N, K, \beta, \mu, m, \delta_{2}\right) \in E_{B_{1}}$, the characteristic values of $B\left(x^{*}, y^{*}\right)$ are a pair of complex conjugate numbers $\lambda_{3}$ and $\lambda_{4}$ with modulus 1 :

$$
\lambda_{3,4}=1+\frac{E\left(\delta_{2}+\delta^{\star}\right)}{2} \mp \frac{i\left(\delta_{2}+\delta^{\star}\right) \sqrt{4 F-E^{2}}}{2} .
$$

Now, we have

$$
\begin{aligned}
\left|\lambda_{3,4}\right| & =\sqrt{q\left(\delta^{\star}\right),} \\
l & =\frac{d|\lambda|}{d \delta^{\star}}=-\frac{E}{2}>0 .
\end{aligned}
$$

When $\delta^{\star}$ varies in limited neighborhood of $\delta^{\star}=0$, let $\theta=\operatorname{Re}(\lambda)=1+\delta_{2} E / 2, \omega=\operatorname{Im}(\lambda)=\delta_{2}\left(\sqrt{4 F-E^{2}} / 2\right)$. Neimark-Sacker bifurcation requires that when $\delta^{\star}=0$, then 
$\bar{\lambda}^{n}, \lambda^{n} \neq 1(n=1,2,3,4)$, which is equivalent to $p(0) \neq$ $0,2,-2,1$. Since $p(0)^{2}-4 q(0)<0$ and $q(0)=1$, therefore $p(0)^{2}<4$; then $p(0) \neq \pm 2$, which follows that

$$
E^{2} \neq 2 F, 3 F
$$

Let

$$
T=\left[\begin{array}{cc}
b_{12} & 0 \\
\theta-b_{11} & -\omega
\end{array}\right] \text {. }
$$

Consider the translation below:

$$
\left[\begin{array}{l}
X \\
Y
\end{array}\right]=T\left[\begin{array}{l}
u \\
v
\end{array}\right]
$$

Taking $T^{-1}$ on both sides of (19), obtain

$$
\left[\begin{array}{l}
u \\
v
\end{array}\right]=\left[\begin{array}{cc}
\theta & -\omega \\
\omega & \theta
\end{array}\right]\left[\begin{array}{l}
u \\
v
\end{array}\right]+\left[\begin{array}{l}
f(u, v) \\
g(u, v)
\end{array}\right]
$$

where

$$
\begin{aligned}
& f(u, v)=b_{13}\left[\left(\theta-b_{11}\right) u^{2}-\omega u v\right], \\
& \begin{aligned}
g(u, v) \\
\quad=-\frac{1}{\omega}\left[b_{13}\left(b_{11}-\theta\right)+b_{12} b_{23}\right] u\left[\left(\theta-b_{11}\right) u-\omega v\right], \\
X=b_{12} u \\
Y=\left(\theta-b_{11}\right) u-\omega v .
\end{aligned}
\end{aligned}
$$

Now

$$
\begin{aligned}
f_{u u} & =2 b_{13}\left(\theta-b_{11}\right), \\
f_{u v} & =-b_{13} \omega, \\
f_{v v} & =0, \\
f_{u u u} & =f_{u u v}=f_{u v v}=f_{v v v}=0, \\
g_{u u} & =-\frac{2}{\omega}\left[b_{13}\left(b_{11}-\theta\right)+b_{12} b_{23}\right]\left(\theta-b_{11}\right), \\
g_{u v} & =b_{12}\left(b_{11}-\theta\right)+b_{12} b_{23}, \\
g_{v v} & =0, \\
g_{u u u} & =g_{u u v}=g_{u v v}=g_{v v v}=0 .
\end{aligned}
$$

According to Neimark-Sacker bifurcation, the discriminatory quantities are given by

$$
\begin{aligned}
\mu_{2}= & -\operatorname{Re}\left[\frac{(1-2 \lambda) \bar{\lambda}^{2}}{1-\lambda} \Phi_{11} \Phi_{20}\right]-\frac{1}{2}\left\|\Phi_{11}\right\|^{2} \\
& -\left\|\Phi_{02}\right\|^{2}+\operatorname{Re}\left(\bar{\lambda} \Phi_{21}\right),
\end{aligned}
$$

where

$$
\begin{aligned}
\Phi_{20} & =\frac{1}{8}\left[\left(f_{u u}-f_{v v}+2 g_{u v}\right)+i\left(g_{u u}-g_{v v}-2 f_{u v}\right)\right], \\
\Phi_{11} & =\frac{1}{4}\left[\left(f_{u u}+f_{v v}\right)+i\left(g_{u u}+g_{v v}\right)\right], \\
\Phi_{02} & =\frac{1}{8}\left[\left(f_{u u}-f_{v v}-2 g_{u v}\right)+i\left(g_{u u}-g_{v v}+2 f_{u v}\right)\right], \\
\Phi_{21} & =\frac{1}{16}\left[\left(f_{u u u}+f_{u v v}+g_{u u v}+g_{v v v}\right)\right. \\
+ & \left.i\left(g_{u u u}+g_{u v v}-f_{u u v}-f_{v v v}\right)\right] .
\end{aligned}
$$

Then we can conclude the following theorem.

Theorem 6. If condition (39) holds, $\mu_{2} \neq 0$, when the parameter $\delta^{\star}$ alters around the limit region of point $(0,0)$, system (2) undergoes a Neimark-Sacker bifurcation at the point $B\left(X^{*}, Y^{*}\right)$. If $\mu_{2}<0$, an attracting invariant closed curve bifurcates from the point $B\left(X^{*}, Y^{*}\right)$ for $\delta^{\star}>0$.

\section{Numerical Simulation}

In this section, we will give the numerical simulations for model (2), which not only illustrate our theoretical analysis but also present the complex, rich, and surprising dynamical behavior. The bifurcation parameters are considered for the following two cases:

(1) varying $\delta$ in the range $4<\delta<7$ and fixing $\beta=0.1$, $r=0.03, d=0.0004, \mu=0.006, m=0.0004, N=10$, and $K=500$;

(2) varying $\delta$ in the range $2.8<\delta<3.6$ and fixing $\beta=$ $0.45, r=6, d=0.6, \mu=0.1, m=0.3, N=1$, and $K=1.25$.

Case 1. For Case $1 \beta=0.1, r=0.03, d=0.0004, \mu=0.006$, $m=0.0004, N=10$, and $K=500$ with fixed point $(0.64,45.2975)$ at the initial value point $(1,1)$. By a simple calculation, we get $\delta_{1}=4.438, \alpha_{1} \neq 0, \alpha_{2}=1.01298931>0$, which established Theorem 5 . From bifurcation diagram (see Figure 1(c)) and the largest Lyapunov exponent of model (2) (see Figure 1(a)), the fixed point of system (2) is stable when $\delta_{1}<4.438$ and unstable with $\delta_{1}=4.438$ and there exists flip bifurcation when $\delta_{1}>4.438$. Largest Lyapunov exponents (Figure 1(a)) correspond to Figure 1(c).

The phase portraits which are associated with Figures 1(a) and 1(c) are displayed in Figure 3. There are the orbits of periods 2,4 , and 8 with $\delta_{1} \in(4.438,5.675)$. Moreover, when $\delta_{1} \in[5.675,7)$, stable period windows and chaotic sets alternate in appearing. For example, there emerge the stable window of period-9, period-7, period-3, period-5, period6 , period-1 orbits. The phase portraits which are associated with Figures $1(\mathrm{~b})$ and $1(\mathrm{~d})$ are displayed in Figure 3. With $\delta_{1}$ increasing, chaos and period-doubling bifurcation take place by turn. Finally, the system enters into chaos. 


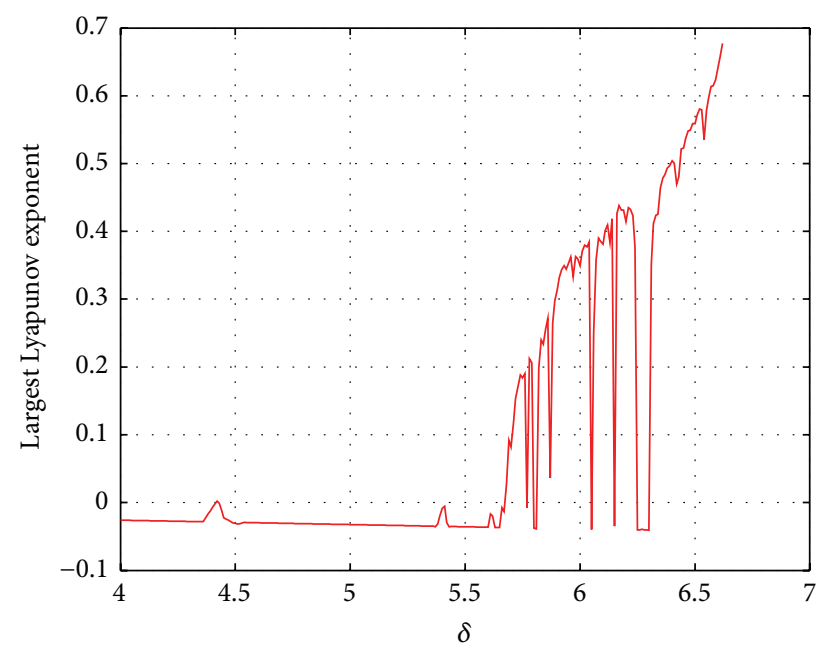

(a)

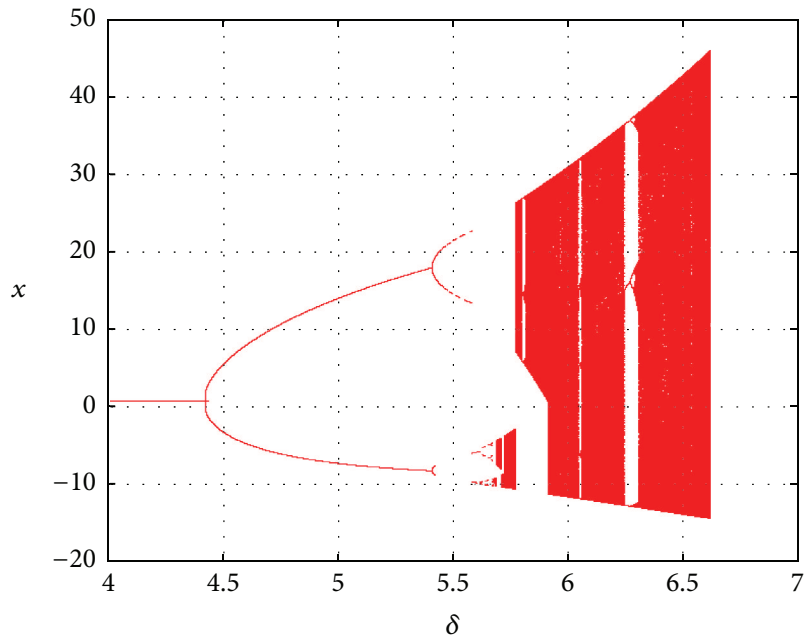

(c)

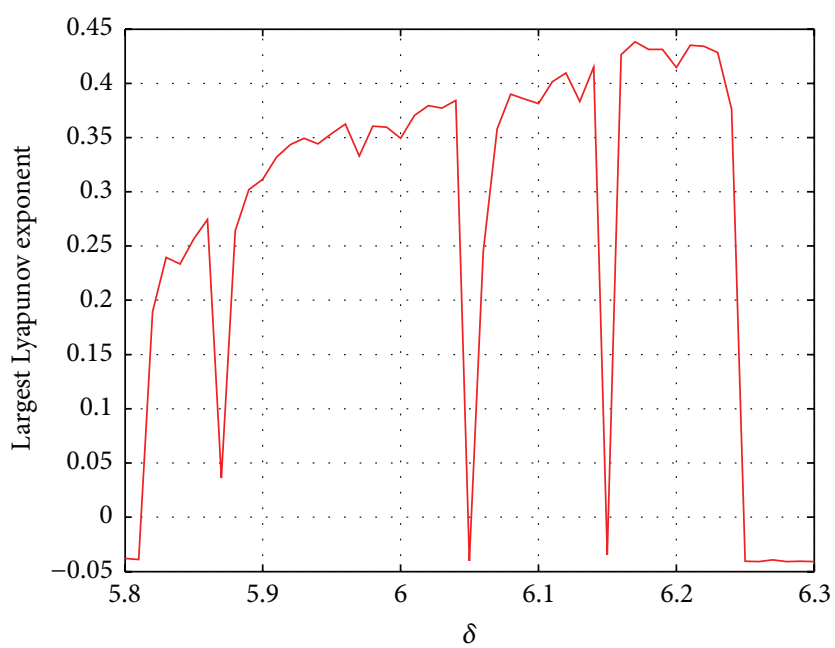

(b)

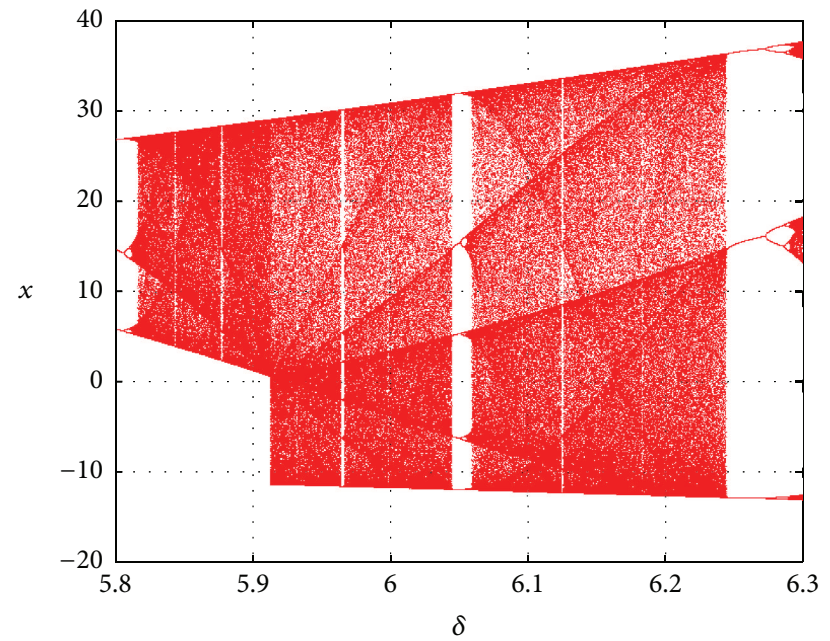

(d)

FIGURE 1: (a) Largest Lyapunov exponents corresponding to (c). (c) Flip bifurcation diagrams of system (2) in $(\delta, x)$ at the fixed point $B(0.64,45.2975)$, and not only the initial value point $(1,1)$, but also $\beta=0.1, r=0.03, d=0.0004, \mu=0.006, m=0.0004, N=10$, and $K=500$; meanwhile, $\alpha_{2}=1.0129893>0$. (b) Largest Lyapunov exponents corresponding to (d). (d) Local amplification of (b) for $\delta \in(5.8,6.3)$.

Case 2. For Case $2 \beta=0.45, r=6, d=0.6, \mu=0.1, m=0.3$, $N=1$, and $K=1.25$. The initial value is $(1,1)$, after a simple calculation for the fixed point $(1.55555556,0.82539683)$, we get $\delta_{2}=1.43694715, \lambda_{3,4}=0.44574894 \mp i 0.89515802$, $\theta=0.44574894, \omega=0.89515802,-E / 2=0.3857143>0$, $\Delta_{2}=E^{2}-4 F=-1.55230772<0, E^{2} \neq 2 F, 3 F, \mu_{2}<0$ (see Figure 2(b)); it established Theorem 6. From Figures 2(a) and 2(c), we observe that when $\delta_{2}<2.96$, the fixed point of map (2) is stable, it loses its stability at $\delta_{2}=2.96$, and there appears an invariant circle when $\delta_{2}$ exceeds 2.96. Figures 2(b) and 2(d) are local amplifications of Figures 2(a) and 2(c) respectively for $\delta_{2} \in(3.25,3.4)$.

The phase portraits which are associated with Figures 1(a) and 1(c) are displayed in Figure 4. Lyapunov exponents are smaller than 0 when $\delta \in(0.25,0.296)$; that is to say, the nonchaotic region is smaller than the chaotic region $(0.296,0.34)$. For $\delta_{2} \in(0.296,0.34)$, some Lyapunov exponents are larger than 0 and some Lyapunov exponents are smaller than 0 , so there exists stable fixed point or stable period windows in the chaotic region. As we all know, Lyapunov exponents are bigger than 0 which implies the existence of chaos. With $\delta_{2}$ increases, circle disappears and period-53, period-34, period-19, period-23, period-19, period-42, period-15, and period-11 orbits appear, attracting chaotic sets (see Figure 4). From Figure 4, we can see that the attractive cycle is smooth in the vicinity of the bifurcation point. However, when $\delta_{2}$ decreases, the cycle inflates gradually and loses its smoothness, and the system enters chaos. This shows that not only the stability changes of system but also the limit cycle occur when system crosses the bifurcation point. 


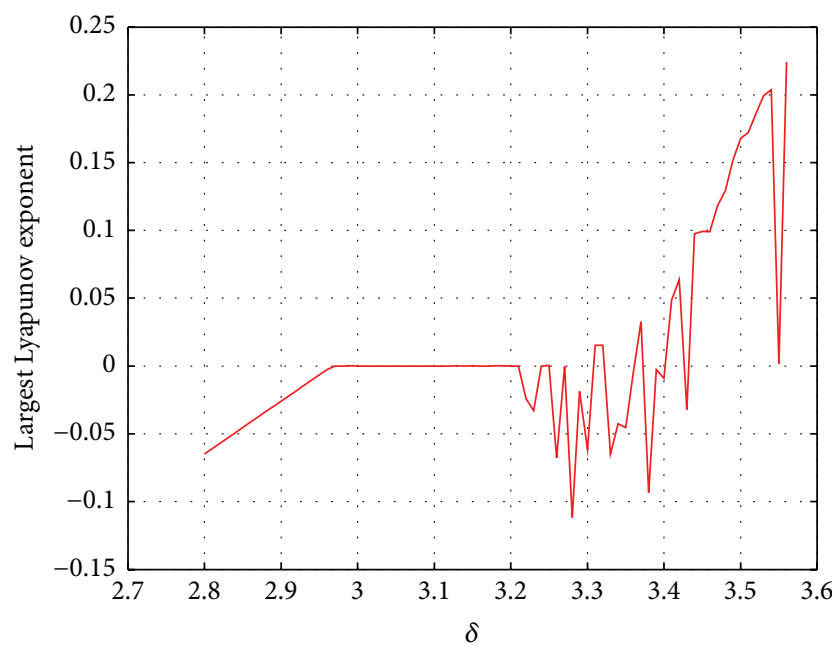

(a)

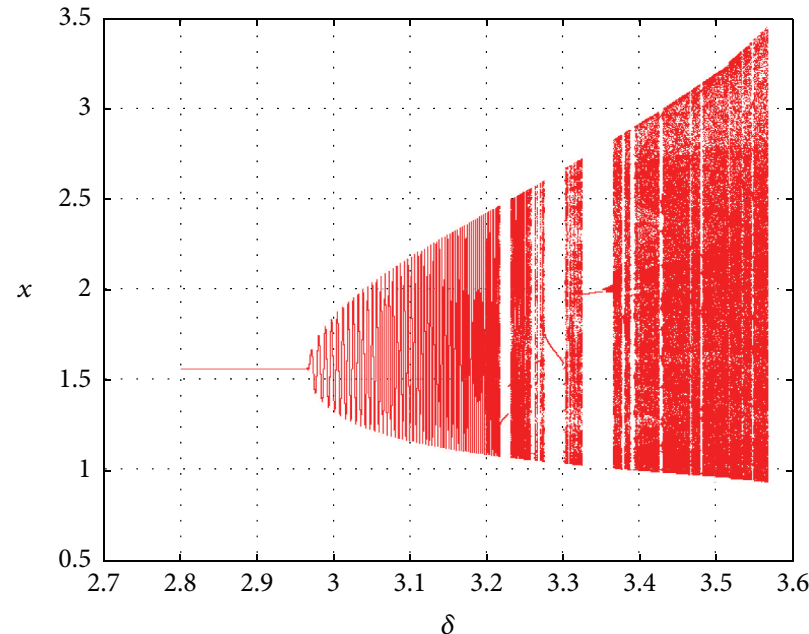

(c)

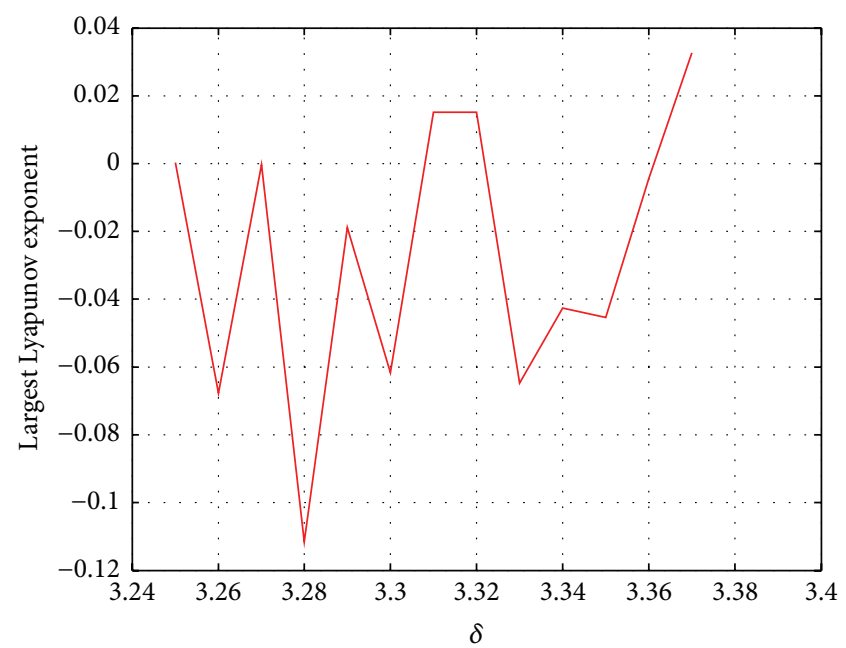

(b)

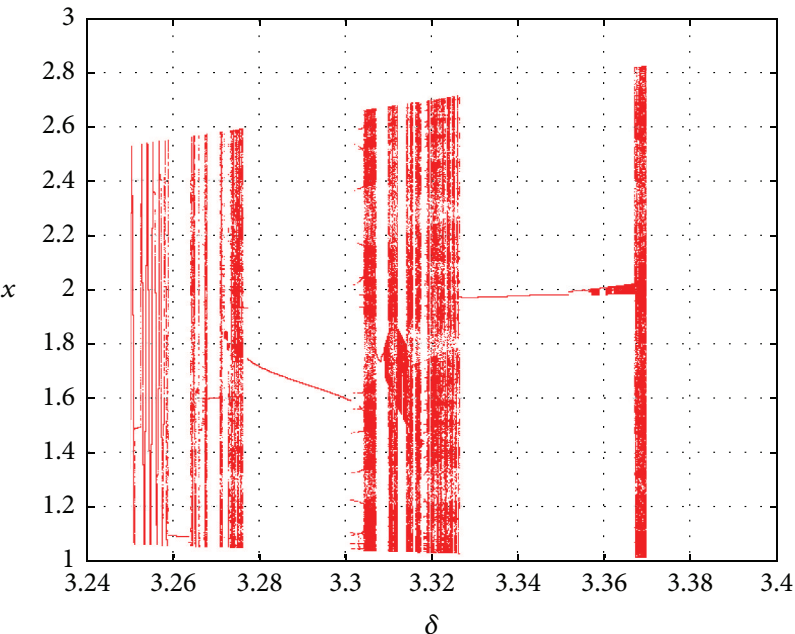

(d)

Figure 2: (a) Largest Lyapunov exponents corresponding to (c). (c) Neimark-Sacker bifurcation diagrams of system (2) in $(\delta$ - $x$ ) plane at the point $B(1.55555556,0.82539683)$, and not only the initial value point $(1,1)$, but also $\beta=0.45, r=6, d=0.6, \mu=0.1, m=0.3, N=1$, and $K=1.25$. (b) Largest Lyapunov exponents corresponding to (d). (d) Local amplification of (b) for $\delta \in(3.25,3.37)$.

\section{Chaos Control}

In this section, a feedback control law as the control force $u_{n}$ is added to (2): the following controlled form of model (2) is

$$
\begin{aligned}
x_{n+1}= & x_{n}+\delta\left[r N\left(1-\frac{N}{K}\right)-\beta \frac{x_{n} y_{n}}{N}-(\mu+m) x_{n}\right] \\
& +u_{n}, \\
y_{n+1}= & y_{n}+\delta\left[\beta \frac{x_{n} y_{n}}{N}-(\mu+d) y_{n}\right], \\
u_{n}= & -k_{1}\left(x_{n}-x^{*}\right)-k_{2}\left(y_{n}-y^{*}\right),
\end{aligned}
$$

where $k_{1}$ and $k_{2}$ are the feedback gain and $\left(x^{*}, y^{*}\right)$ is the positive point of model (2). The Jacobian matrix $J$ of controlled system (46) is given by

$$
J=\left[\begin{array}{cc}
a_{11}-k_{1} & a_{12}-k_{2} \\
a_{21} & a_{22}
\end{array}\right],
$$

where $a_{11}, a_{12}, a_{21}, a_{22}$ are given in (17). The characteristic equation of (48) is

$$
\begin{gathered}
\lambda^{2}-\left(a_{11}+a_{22}-k_{1}\right) \lambda+a_{22}\left(a_{11}-k_{1}\right) \\
-a_{21}\left(a_{12}-k_{2}\right)=0 .
\end{gathered}
$$

Let $\lambda_{1}$ and $\lambda_{2}$ be the two roots of (49); then

$$
\begin{aligned}
\lambda_{1}+\lambda_{2} & =a_{11}+a_{22}-k_{1}, \\
\lambda_{1} \lambda_{2} & =a_{22}\left(a_{11}-k_{1}\right)-a_{21}\left(a_{12}-k_{2}\right) .
\end{aligned}
$$

The lines of marginal stability are determined by solving the equation of $\lambda_{1}= \pm 1$ and $\lambda_{1} \lambda_{2}=1$.

These conditions guarantee that the eigenvalues $\lambda_{1}$ and $\lambda_{2}$ have modulus less than 1 . 
Discrete Dynamics in Nature and Society

9
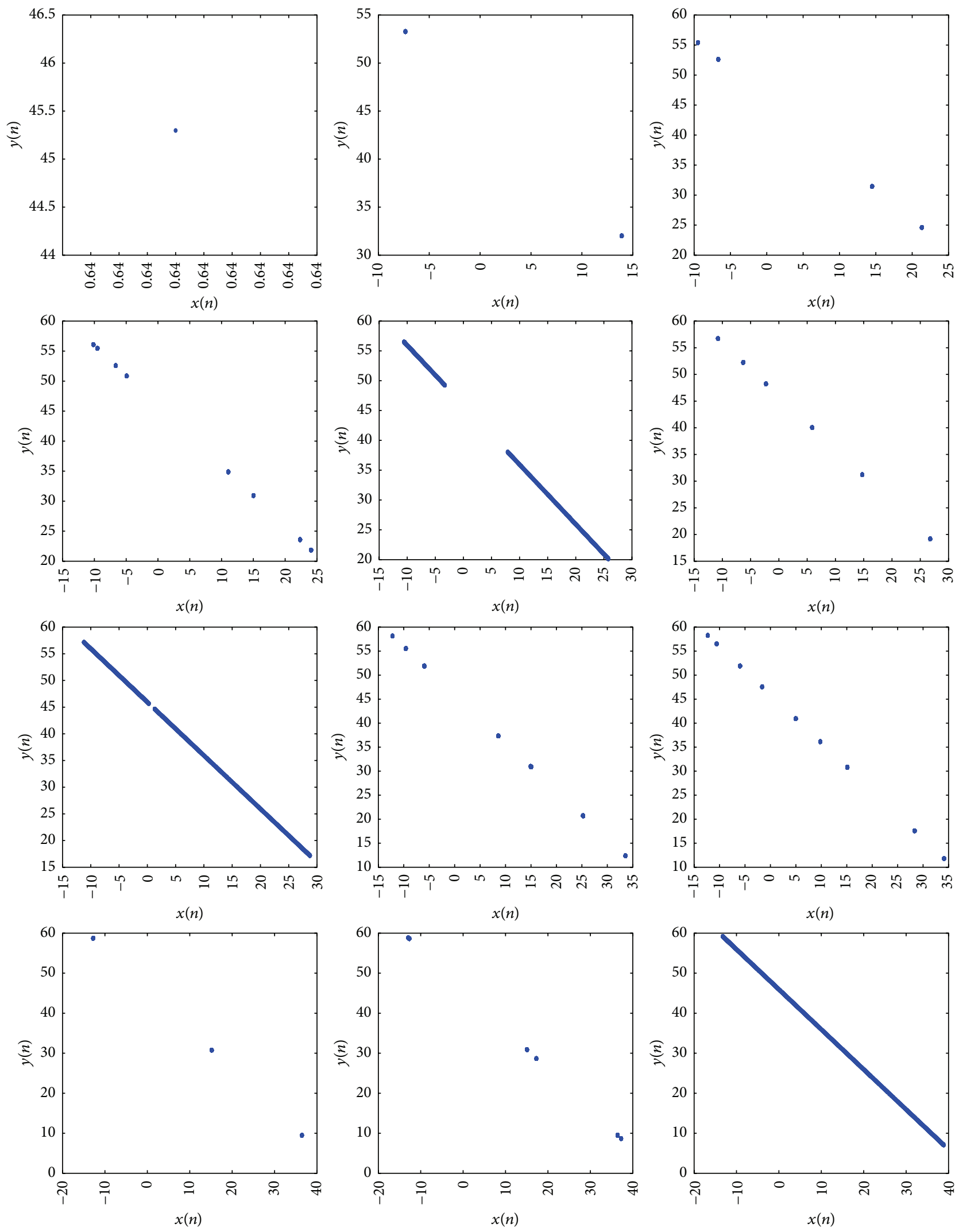

Figure 3: Phase portrait of system (2) at the initial value $(1,1) ; \beta=0.1, r=0.03, d=0.0004, \mu=0.006, m=0.0004, \delta=4.3, \delta=5, \delta=5.5$, $\delta=5.66, \delta=5.75, \delta=5.8, \delta=5.9, \delta=6.125, \delta=6.15, \delta=6.25, \delta=6.285$, and $\delta=6.35$. 

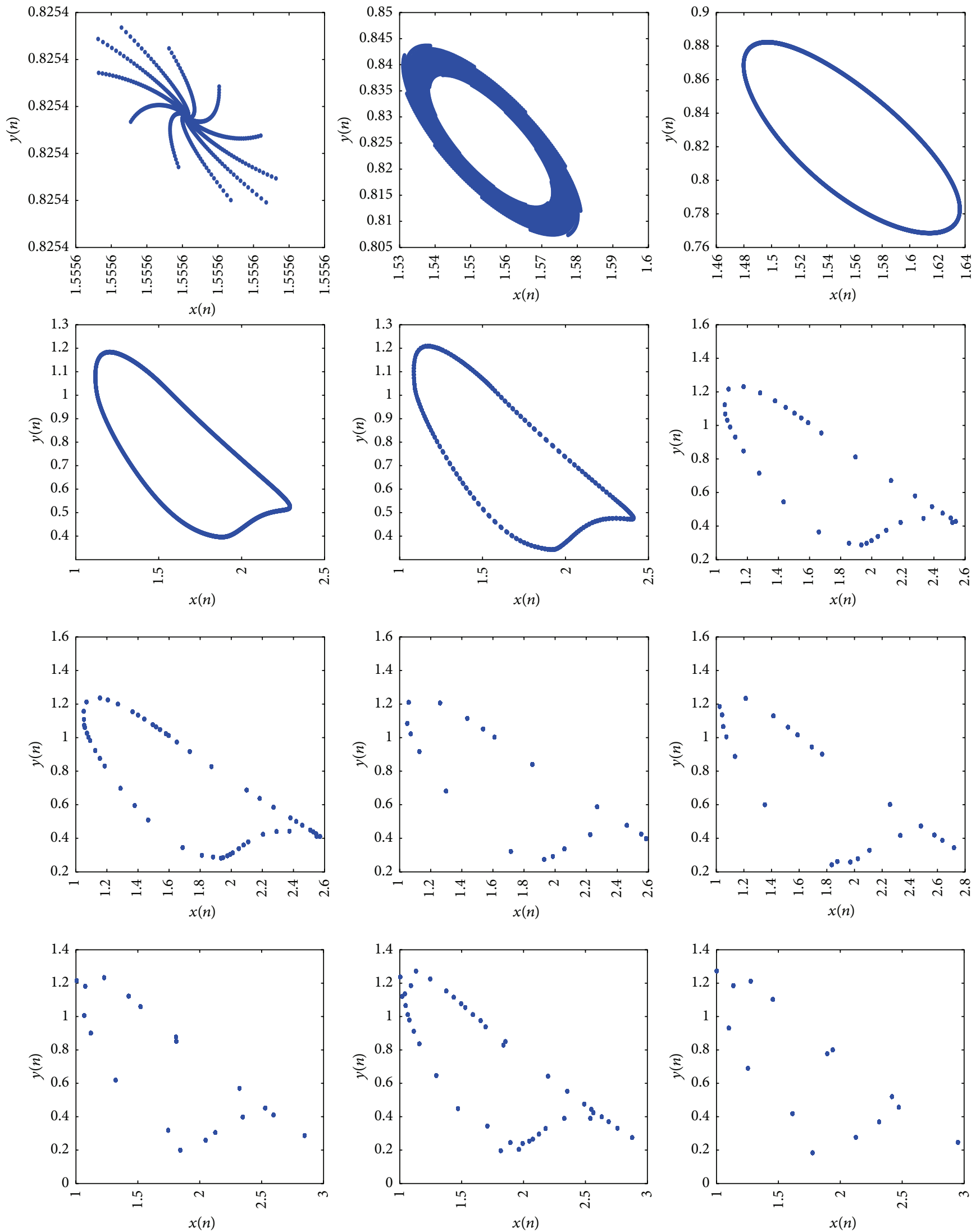

FIgUre 4: Continued. 

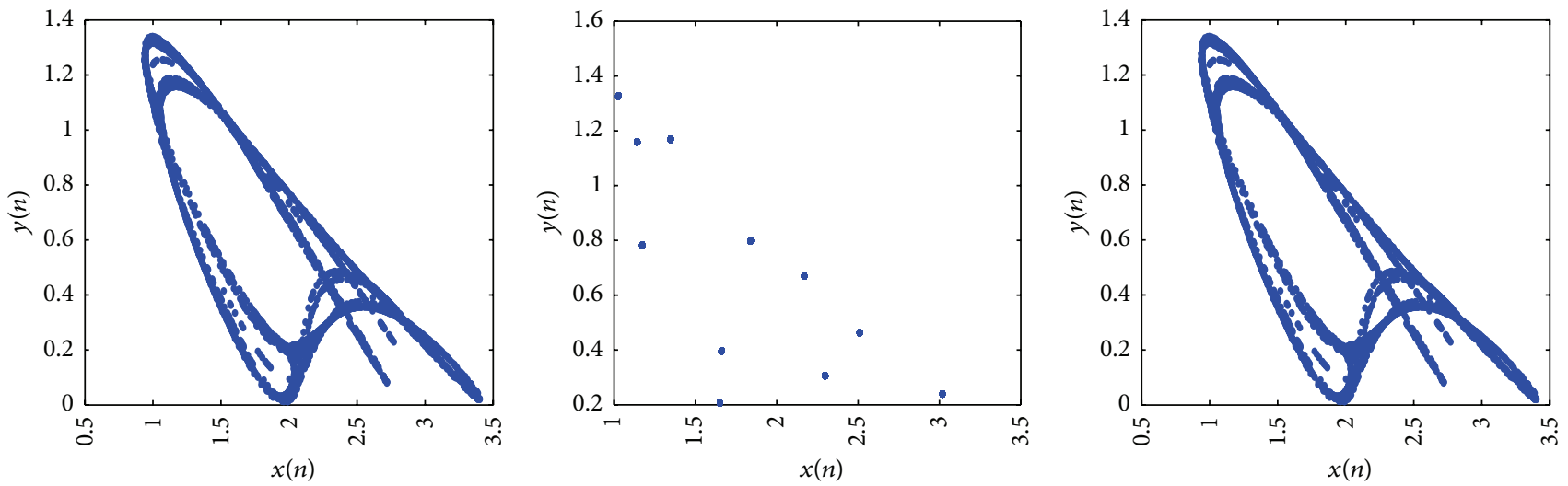

Figure 4: Neimark-Sacker bifurcation phase portrait of system (2) with the initial value point $(1,1)$ and $\delta=2.955, \delta=2.967, \delta=2.97$, $\delta=3.15, \delta=3.2, \delta=3.261, \delta=3.2685, \delta=3.28, \delta=3.33, \delta=3.379, \delta=3.391, \delta=3.429, \delta=3.54, \delta=3.5485$, and $\delta=3.56$, respectively.

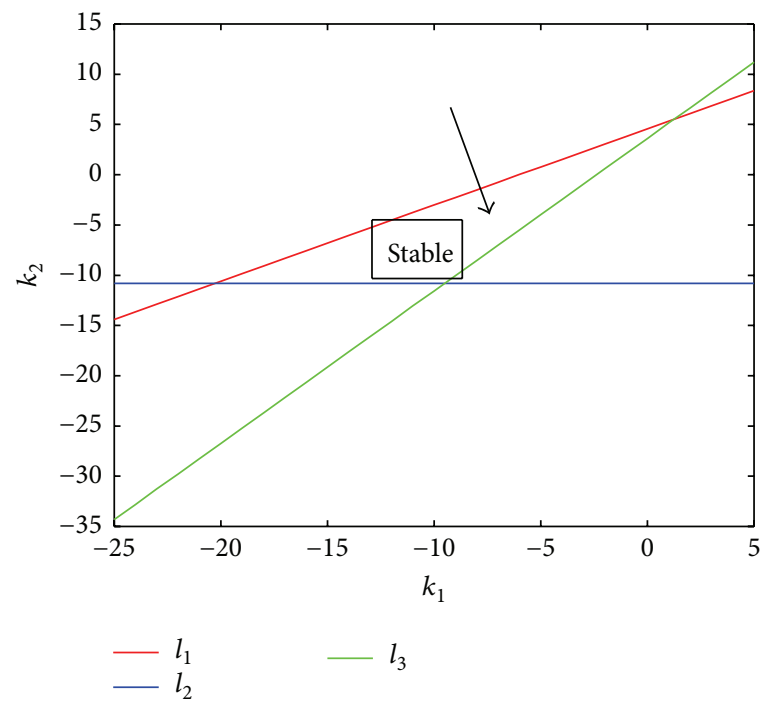

(a)

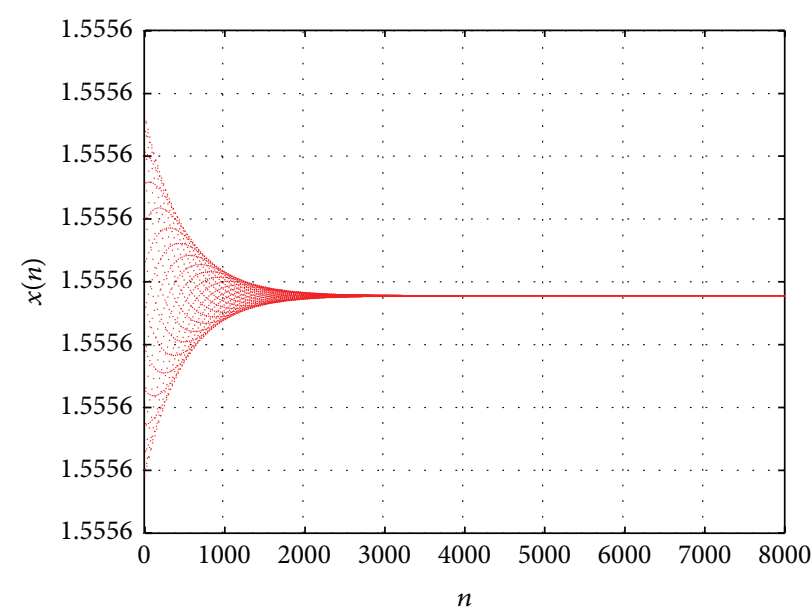

(b)

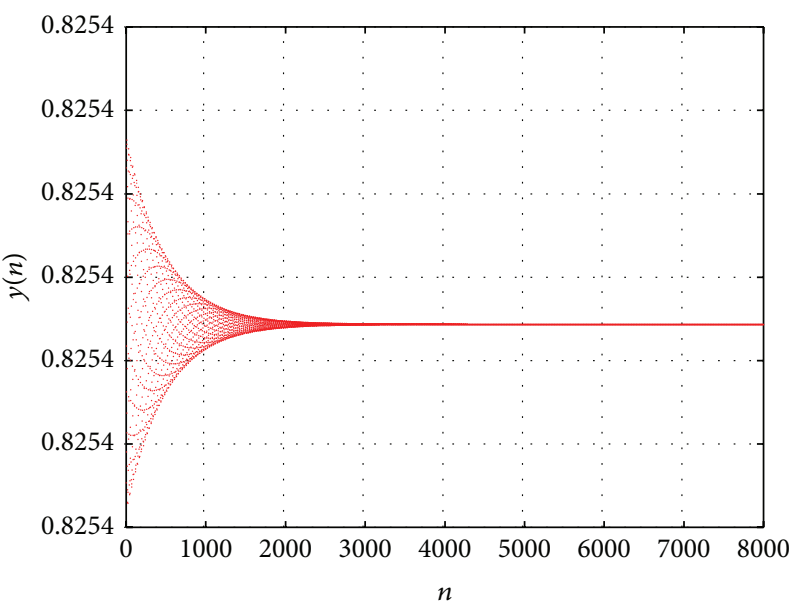

(c)

Figure 5: (a) The bounded region for the eigenvalues of control model (48) and (49) in the $\left(k_{1}, k_{2}\right)$ plane for $\beta=0.045, r=6, d=0.6$, $\mu=0.1, m=0.3, N=1, K=1.25$, and $\delta=3.55$. (b) The time responses for the state $x$ of control model (48) and (49) in the ( $n, x)$ plane for $\beta=0.045, r=6, d=0.6, \mu=0.1, m=0.3, N=1, K=1.25, \delta=3.556, k_{1}=0.1$, and $k_{2}=-0.3415$. The initial value point (1,1). (c) The time responses for the state $y$ of control model $(48)$ and $(49)$ in the $(n, y)$ plane. 
Assume that $\lambda_{1} \lambda_{2}=1$, and from (51) we have

$l_{1}: k_{1} a_{22}-k_{2} a_{21}=a_{11} a_{22}-a_{12} a_{21}-1$.

Assume that $\lambda_{1}=1$, and from (50) and (51) we have

$l_{2}: k_{1}\left(1-a_{22}\right)-k_{2} a_{21}=a_{11}+a_{22}-1-a_{11} a_{22}+a_{12} a_{21}$.

Assume that $\lambda_{1}=-1$, and from (50) and (51) we have

$l_{3}: k_{1}\left(1+a_{22}\right)-k_{2} a_{21}=a_{11}+a_{22}+1+a_{11} a_{22}-a_{12} a_{21}$.

The stable eigenvalues lie within a triangular region by lines $l_{1}, l_{2}$, and $l_{3}$ (see Figure 5(a)).

We have performed some numerical simulations to see how the state feedback method controls the unstable fixed point. Parameter values are fixed as $\beta=0.45, r=6, d=0.6$, $\mu=0.1, m=0.3, N=1, K=1.25$, and $\delta=3.55$. The initial value is $(1,1)$, and the feedback gain $k_{1}=0.2, k_{2}=-0.34$. It is shown in Figures 5(b) and 5(c) that a chaotic trajectory is stabilized at the fixed point (1.55555556, 0.82539683).

\section{Discussion}

The dynamics of discrete SI epidemic model, which has been obtained by the forward Euler scheme, is investigated in detail. By using the center manifold theorem and bifurcation theorem in the closed first quadrant $R_{+}^{2}$, the specific conditions for the existence of flip bifurcation and NeimarkSacker bifurcation have been derived. Moreover, numerical simulation not only presents our theoretical analysis but also exhibits rich and complex dynamical behavior existing in the case of the windows of period-1, period-3, period5 , period-6, period-7, period-9, period-11, period-15, period19, period-23, period-34, period-42, and period-53 orbits. Meanwhile, there appears the cascade of period-doubling 2, 4, 8 bifurcation and chaos sets from the fixed point. These results show that the discrete model has more richer dynamics compared with the continuous model. The computations of the largest Lyapunov exponents more than 0 confirm the chaotic behaviors of system (2). Specifically, the chaotic orbits at an unstable fixed point are stabilized by using the feedback control method. However, in this paper, respect to the interpretation of these results with sound meaning of general disease dynamics and implications for disease control still need further researches. In our future work, we expect to obtain some more results based on real data from known epidemic disease to illustrate the validity of our theoretic results, such as how to predict the occurrence of disease in which way study global stability, limit cycle, bifurcation, chaos, strange attractors, hidden attractor impact on dynamics of disease, and so on.

\section{Conflict of Interests}

The authors declare that there is no conflict of interests regarding the publication of this paper.

\section{Acknowledgments}

This work was supported by National Natural Science Foundation of China (Grant no. 61261044) and the Education Department Foundation of Henan Province (Grant no. 15A110046). And the authors would like to thank the suggestions of the anonymous referees in their original paper.

\section{References}

[1] D. J. D. Earn, P. Rohani, B. M. Bolker, and B. T. Grenfell, "A single epidemic model with surpising dynamics," Mathematical Biosciences and Engineering, vol. 2, pp. 133-152, 2005.

[2] E. Sáez, E. Stange, I. Szántó, E. González-Olivares, and M. Falconi, "Chaotic dynamics and coexistence in a three species interaction model," International Journal of Biomathematics, vol. 8, no. 2, Article ID 1550022, 25 pages, 2015.

[3] J. Wei and J.-A. Cui, "Dynamics of SIS epidemic model with the standard incidence rate and saturated treatment function," International Journal of Biomathematics, vol. 5, no. 3, Article ID 1260003, 18 pages, 2012.

[4] L. Zhou and M. Fan, "Dynamics of an SIR epidemic model with limited medical resources revisited," Nonlinear Analysis: Real World Applications, vol. 13, no. 1, pp. 312-324, 2012.

[5] L. Wang, L. Chen, and J. J. Nieto, "The dynamics of an epidemic model for pest control with impulsive effect," Nonlinear Analysis. Real World Applications, vol. 11, no. 3, pp. 1374-1386, 2010.

[6] P. Das, Z. Mukandavire, C. Chiyaka, A. Sen, and D. Mukherjee, "Bifurcation and chaos in S-I-S epidemic model," Differential Equations and Dynamical Systems, vol. 17, no. 4, pp. 393-417, 2009.

[7] S. Sharma and G. P. Samanta, "A Leslie-Gower predator-prey model with disease in prey incorporating a prey refuge," Chaos, Solitons \& Fractals, vol. 70, pp. 69-84, 2015.

[8] S. Sharma and G. P. Samanta, "A ratio-dependent predator-prey model with Allee effect and disease in prey," Journal of Applied Mathematics and Computing, vol. 47, no. 1-2, pp. 345-364, 2015.

[9] T. Zhang and Z. Teng, "Global behavior and permanence of SIRS epidemic model with time delay," Nonlinear Analysis. Real World Applications, vol. 9, no. 4, pp. 1409-1424, 2008.

[10] X.-Z. Li, W.-S. Li, and M. Ghosh, "Stability and bifurcation of an SIS epidemic model with treatment," Chaos, Solitons \& Fractals, vol. 42, no. 5, pp. 2822-2832, 2009.

[11] A. Suryanto, W. M. Kusumawinahyu, I. Darti, and I. Yanti, "Dynamically consistent discrete epidemic model with modified saturated incidence rate," Computational \& Applied Mathematics, vol. 32, no. 2, pp. 373-383, 2013.

[12] H. Jansen and E. H. Twizell, "An unconditionally convergent discretization of the SEIR model," Mathematics and Computers in Simulation, vol. 58, no. 2, pp. 147-158, 2002.

[13] J. Liu, B. Peng, and T. Zhang, "Effect of discretization on dynamical behavior of SEIR and SIR models with nonlinear incidence," Applied Mathematics Letters, vol. 39, pp. 60-66, 2015.

[14] M. Sekiguchi, "Permanence of a discrete SIRS epidemic model with time delays," Applied Mathematics Letters, vol. 23, no. 10, pp. 1280-1285, 2010.

[15] Y. Muroya, A. Bellen, Y. Enatsu, and Y. Nakata, "Global stability for a discrete epidemic model for disease with immunity and latency spreading in a heterogeneous host population," Nonlinear Analysis. Real World Applications, vol. 13, no. 1, pp. 258-274, 2012. 
[16] Y. Muroya, Y. Nakata, G. Izzo, and A. Vecchio, "Permanence and global stability of a class of discrete epidemic models," Nonlinear Analysis: Real World Applications, vol. 12, no. 4, pp. 2105-2117, 2011.

[17] D. Hu and H. Cao, "Bifurcation and chaos in a discrete-time predator-prey system of Holling and Leslie type," Communications in Nonlinear Science and Numerical Simulation, vol. 22, no. 1-3, pp. 702-715, 2015.

[18] G. Zhang, Y. Shen, and B. Chen, "Bifurcation analysis in a discrete differential-algebraic predator-prey system," Applied Mathematical Modelling, vol. 38, no. 19-20, pp. 4835-4848, 2014.

[19] H. Cao, Y. Zhou, and Z. Ma, "Bifurcation analysis of a discrete SIS model with bilinear incidence depending on new infection," Mathematical Biosciences and Engineering, vol. 10, no. 5-6, pp. 1399-1417, 2013.

[20] J. Dhar, H. Singh, and H. S. Bhatti, "Discrete-time dynamics of a system with crowding effect and predator partially dependent on prey," Applied Mathematics and Computation, vol. 252, pp. 324-335, 2015.

[21] X. Liu and D. Xiao, "Complex dynamic behaviors of a discretetime predator-prey system," Chaos, Solitons \& Fractals, vol. 32, no. 1, pp. 80-94, 2007.

[22] X.-W. Chen, X.-L. Fu, and Z.-J. Jing, "Dynamics in a discretetime predator-prey system with Allee effect," Acta Mathematicae Applicatae Sinica, vol. 29, no. 1, pp. 143-164, 2013.

[23] X.-W. Chen, X.-L. Fu, and Z.-J. Jing, "Complex dynamics in a discrete-time predator-prey system without Allee effect," Acta Mathematicae Applicatae Sinica-English Series, vol. 29, no. 2, pp. 355-376, 2013.

[24] X. Wang, M. Peng, R. Cheng, and J. Yu, "Stability analysis and rich oscillation patterns in discrete-time FitzHugh-Nagumo excitable systems with delayed coupling," Nonlinear Dynamics, vol. 78, no. 3, pp. 2085-2099, 2014.

[25] Y. Guo, "Chaos and bifurcation in the space-clamped FitzHughNagumo system," Chaos, Solitons \& Fractals, vol. 21, no. 4, pp. 943-956, 2004.

[26] Y. Chow and S. R.-J. Jang, "Dynamics of a system of three interacting populations with Allee effects and stocking," Journal of Difference Equations and Applications, vol. 21, no. 4, pp. 336359, 2015.

[27] Z. He and X. Lai, "Bifurcation and chaotic behavior of a discretetime predator-prey system," Nonlinear Analysis. Real World Applications, vol. 12, no. 1, pp. 403-417, 2011.

[28] Z. Jing, Y. Chang, and B. Guo, "Bifurcation and chaos in discrete FitzHugh-Nagumo system," Chaos, Solitons \& Fractals, vol. 21, no. 3, pp. 701-720, 2004. 


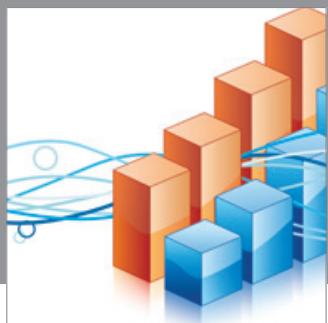

Advances in

Operations Research

mansans

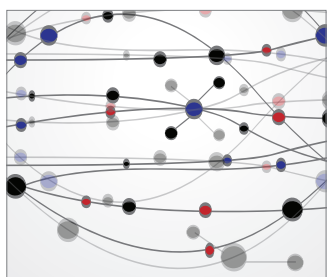

The Scientific World Journal
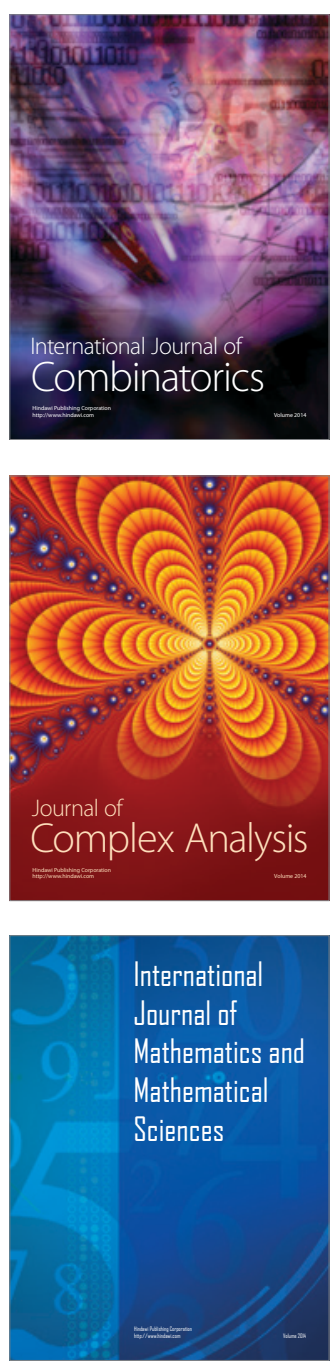
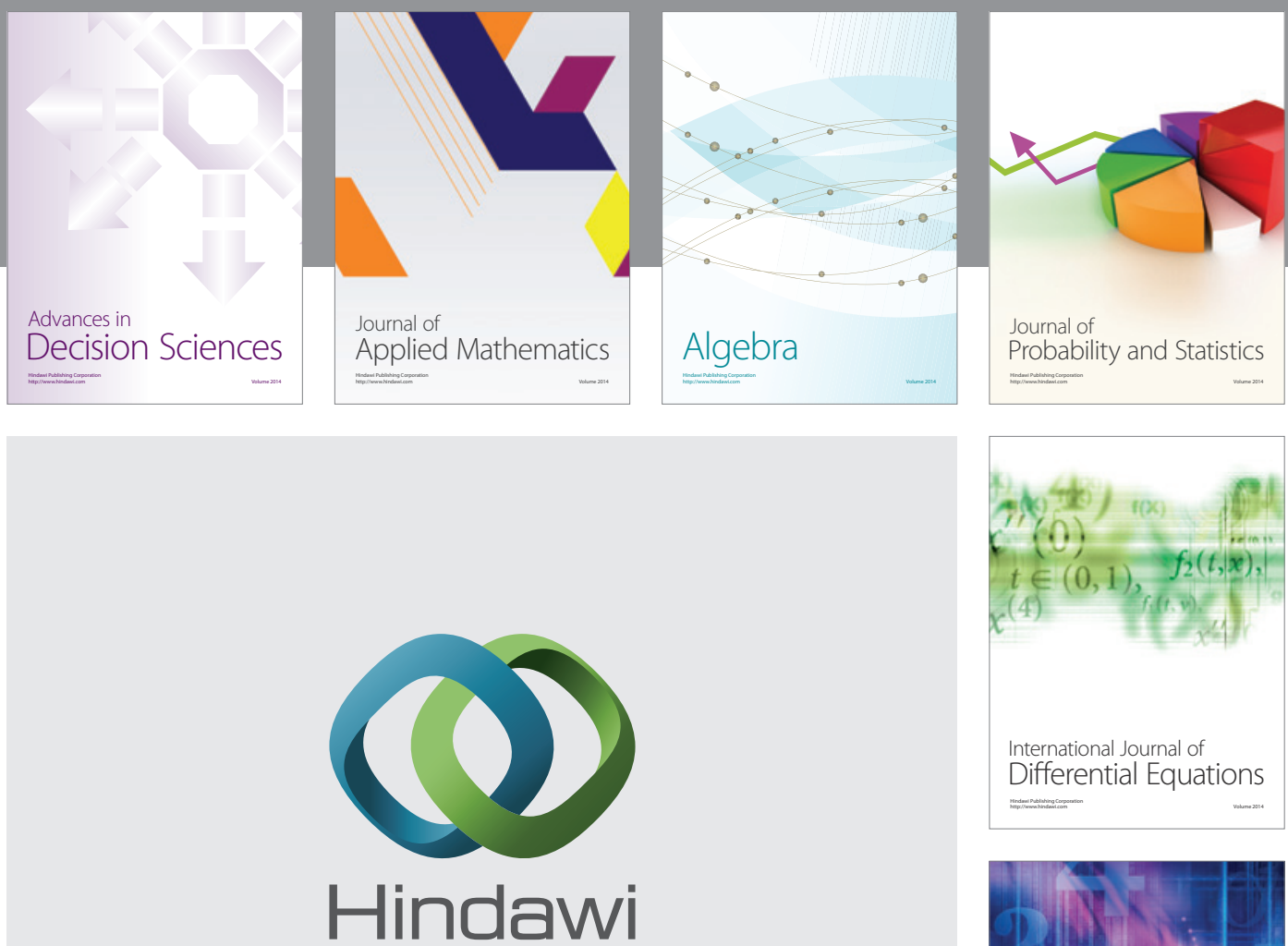

Submit your manuscripts at http://www.hindawi.com
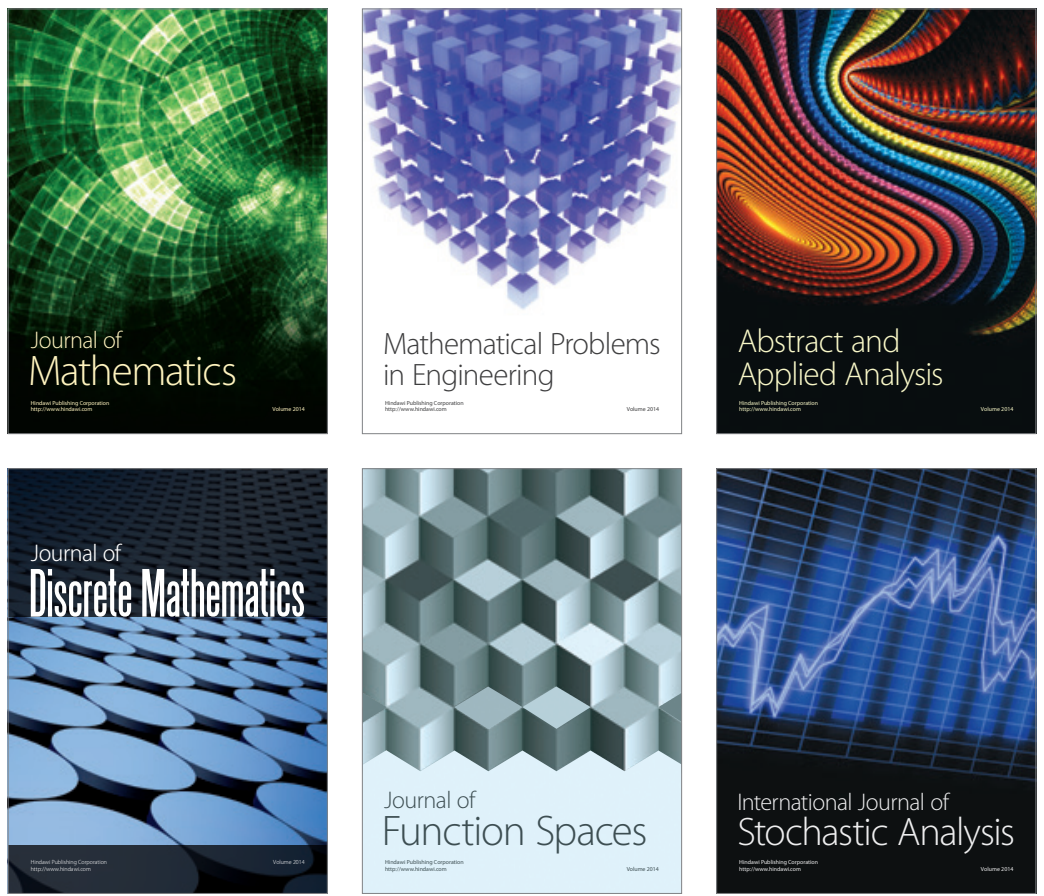

Journal of

Function Spaces

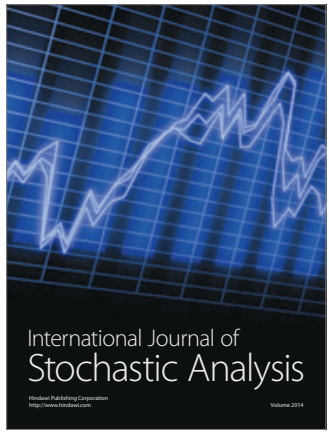

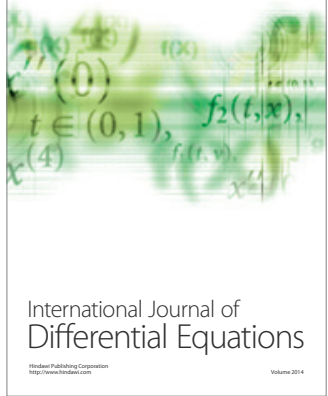
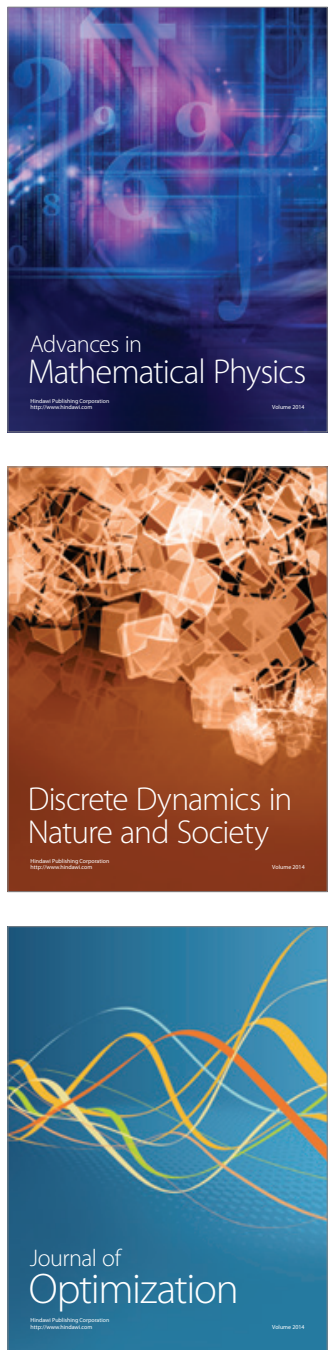